\title{
Environmental stress influences mitochondrial metabolism in vascular cells: consequences for angiogenesis
}

\author{
T Scott Bowen and Stuart Egginton \\ School of Biomedical Sciences, University of Leeds, Leeds, UK \\ Correspondence should be addressed to S Egginton: S.Egginton@leeds.ac.uk
}

\begin{abstract}
While the important and varied roles that vascular cells play in both health and disease is well recognised, the focus on potential therapeutic targets continually shifts as new players emerge. Here, we outline how mitochondria may be viewed as more than simply energygenerating organelles, but instead as important sentinels of metabolic health and effectors of appropriate responses to physiological challenges.
\end{abstract}

\author{
Key Words \\ - capillary \\ - endothelium \\ - ROS \\ - signalling
}

\section{Angiogenesis}

Angiogenesis, the growth of new blood vessels from an existing capillary bed (de novo creation of capillaries, neovascularisation, is a distinct process), is not only essential in the maintenance of tissue/organ health, but also intimately involved in the development of many diseases. This process is commonly viewed as proliferation and migration of endothelial cells (ECs) towards chemical signals (angiogenic growth factors) within the extracellular matrix, although variations on this canonical pathway have been described (1). ECs line all blood vessels and form their smallest entity (the capillaries) that act as the diffusive barrier to $\mathrm{O}_{2}$ and nutritive exchange at the blood/ tissue interface. The growth of new capillaries will extend microcirculatory transport and metabolic capacity, but by nature ECs are one of the most quiescent cell types in the body. As such ECs require a strong signal to stimulate release from contact-inhibition and low turnover rate in order to promote angiogenesis. An effective angiogenic signal, therefore, has to overcome a potent drive for stability in this tubular monolayer of cells. In healthy individuals angiogenesis forms an essential component of, for example, the female reproductive tract cycle, wound repair, and adaptive remodelling induced by exercise training (1). It is recognised that tight control over the angiogenic process is due to tonic influences of both pro- and anti-angiogenic factors, with an altered balance promoting either expansion or rarefaction of the capillary bed $(1,2)$. However, dysfunctional feedback control results in abnormal blood vessel growth, which may be excessive or insufficient depending on the relative strength of stimuli influencing angioadaptation in response to humoral factors and the local environment. Capillaries are much more than a mere physical conduit for erythrocytes delivering $\mathrm{O}_{2}$ to respiring tissues; they are crucial for the removal of waste products, contribute to acid/base and iono/osmo regulation, and impact immune reaction. By virtue of their huge surface area the endothelium is an important interface between systemic hormonal signalling and tissue metabolic responses. Consequently, endothelial dysfunction is known to make https://vb.bioscientifica.com https://doi.org/10.1530/VB-19-0018 (c) 2019 The authors Published by Bioscientifica Ltd

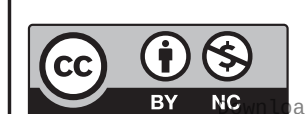

This work is licensed under a Creative Commons Attribution-NonCommercial 4.0 International License. ded from Bioscientifica.com at 04/26/2023 10:47:51AM 
Table 1 Summary of the vascular mitochondrial responses/signals linked to a subsequent angiogenic response and the putative stressors involved.

\begin{tabular}{llc}
\cline { 1 - 1 } Mitochondrial response/signal & & Impact on angiogenesis \\
\cline { 1 - 1 } Biogenesis & & + \\
Shift in fuel oxidation & & - \\
Elevated ROS & & + \\
Antioxidant capacity & - \\
Fission/fusion imbalance & $?$ \\
Oxidative capacity & $?$ \\
Intrinsic respiratory function &
\end{tabular}

Stressor(s)

Hypoxia; caloric restriction; drugs Hyperglycaemia; diabetes Ageing; disease; hypoxia

Exercise; drugs Hyperglycaemia; IR injury Exercise; ageing; nitric oxide Exercise

\begin{tabular}{c}
\hline Reference $(\mathrm{s})$ \\
$(4,16,17)$ \\
$(19,22,23)$ \\
$(14,15,22,23,25)$ \\
$(4,11,12,13,28,29,30)$ \\
$(15)$ \\
$(11,12,14,15)$ \\
$(11)$
\end{tabular}

a major contribution to the aetiology and maintenance of multiple disorders, including those associated with serious risk of mortality and/or level of morbidity, such as cancer, cardiovascular disease, diabetes, stroke, agerelated blindness, psoriasis, rheumatoid arthritis (3) (see also Table 1).

\section{Role of mitochondria in the vasculature}

An important role of EC in the microcirculation is to sense disturbances in physiological set-points in organ systems, such as reacting to changes in haemodynamic forces following altered tissue perfusion (e.g. act as a mechanotransducer for elevated shear stress during skeletal muscle functional hyperaemia) or to local paracrine growth factor production (e.g. act as a chemotransducer during endometrial hypertrophy or tumour progression). There is clearly great incentive to develop pro-angiogenic therapies and anti-angiogenic treatments, but many current approaches need refinement, with combination angiotherapies likely required to provide better efficacy and less toxicity (4). Alternative approaches are therefore required. Understanding the role of EC in the microcirculation, and in angiogenesis in particular, may help focus attention on profitable routes of enquiry including the role of endothelial cell metabolism (2). Hypoxia is thought to be a key driver for vascular remodelling, with observations of oxygen sensing at the capillary level (5) providing a plausible HIF1 $\alpha$-VEGF route linking supply and demand. Mitochondrial function is an important, but underutilised target for improving vascular function, including regulation of vascular tone by activation of mitochondrial $\mathrm{K}_{\mathrm{ATP}}$ channels or ROS production within vascular smooth muscle (6). More recently, a key role has also been suggested for the mitochondria in EC (7). Given their location adjacent to the source of oxygen delivery, it may seem paradoxical that the mitochondrial content in EC is relatively modest: $2-6 \%$ of cytoplasm volume ( $c f$. $32 \%$ in cardiomyocytes (2)).
Further, it appears that energy requirement of EC mitochondria is relatively low compared to myocytes and other cell types that they support (e.g. via transport of nutrients). Indeed, EC are generally fuelled by glycolytic (anaerobic) metabolism, where up to $80 \%$ of ATP is generated by oxygen-independent energy flux (2). Low mitochondrial content is therefore consistent with a role in regulating signalling responses to environmental cues rather than energy production per se (8), however mitochondrial respiration is still necessary for angiogenesis as inhibition of respiratory chain complex III impairs proliferation (with no change in migration) of EC in vitro following a decrease in the $\mathrm{NAD}^{+} / \mathrm{NADH}$ ratio (9). Communication among organelles is, of course, dependent on location. Regulation of fusion and fission will affect mitochondrial distribution and hence potential function: in coronary arterioles they are anchored to the cytoskeleton which facilitates flow-mediated dilatation (10), while hypoxia invokes mitochondrial redistribution around nuclei of pulmonary artery EC (11). Although the importance of EC dysfunction is well known (12, 13), there are many potential roles for mitochondria in vascular responses to various stressors (Table 1 ); we choose to highlight a few of many in this short review.

\section{Interventions impacting vascular mitochondrial signalling}

Various approaches have been established to modulate vascular-located mitochondria and their signalling/ metabolism (Table 1). However, most interventions seem to largely impact vascular mitochondrial signalling properties (unsurprisingly given the low content found within EC), which are especially sensitive to exercise, hypoxia, nutrition, and pharmacological treatments. In addition, other conditions have provided key insight into how normal mitochondrial function in vascular cells becomes disrupted with ageing and/or disease. What remains clear is that modulating mitochondrial properties

This work is licensed under a Creative Commons Attribution-NonCommercial 4.0 International License. 
can have important consequences for subsequent vascular remodelling, which offers a novel therapeutic target worthy of future focus. While the following may also be relevant to perivascular cells, for example, pericyte production of ROS induced by oxidised LDL (14), in the interest of space we concentrate on EC responses per se.

\section{Exercise}

Exercise training is well known to benefit vascular function (15), with mitochondrial signalling implicated to play a key role. Both acute and chronic exercise regimes have important effects on both mitochondrial signalling and morphology/function. Endurance training stimulates improvements in mitochondrial oxidative capacity, as measured from in situ isolated mouse arteries, inducing higher levels of mitochondrial respiration (complex I and II) alongside improving coupling efficiency, but without parallel increases in mitochondrial content (as often seen in other tissues such as skeletal muscle) (16). As such, these data suggest intrinsic mitochondrial function may be more sensitive and of greater importance for driving exercise-related vascular benefits than mitochondrial content per se. Moreover, as complexes I and III are considered to be the main sources of mitochondrial ROS, it is interesting to note that functional improvements were specific to these sites (16). Many of the exerciserelated benefits seem to be consequent to activation of the key nuclear transcriptional co-activator PGC- $1 \alpha$, which is upregulated in vascular tissue following exercise training alongside higher eNOS levels and lower ROS production $(16,17)$. In vitro experiments have confirmed that EC-specific overexpression of PGC- $1 \alpha$ activates the anti-oxidative enzyme transcription network to lower mitochondrial-derived ROS (18). Thus, exercise training seems to improve vascular function by modulating eNOS activity in a redox-sensitive manner, which may be driven by improvements in intrinsic EC mitochondrial function. Further studies are warranted to clarify this issue.

\section{Ageing and disease}

Ageing and disease are both closely related to impaired vascular function. Assessment of feed arteries has shown ageing lowers mitochondrial respiration and this is paralleled by higher mitochondrial ROS generation (19). While data clearly show mitochondrial ROS production is intimately involved in vascular dysfunction, the underlying signalling cascades and how mitochondrial function impacts angiogenesis remain poorly understood.

\section{Ischaemia/inflammation}

One clue may come from the response to tissue ischaemia, where a low $\mathrm{PO}_{2}$ impairs mitochondrial respiration, favouring ROS production and hence EC dysfunction. In atherosclerosis, decreased vascular tone regulation is linked to disturbed NO production, which in turn promotes oxidative stress, platelet aggregation and leukocyte adhesion (20). EC mitochondria respond to hypoxia-induced VEGF with increased biogenesis via AKT-dependent signalling, resulting in increased vascular branching (21), a process reversed by inhibiting biogenesis via the silencing of SIRTUIN1 (22). As SIRTUIN3 is critical for EC glycolysis and angiogenesis, deficiency predisposes to coronary endothelial dysfunction and increases the risk for developing heart failure (23). Fatty acid oxidation (FAO) is essential for EC proliferation during angiogenesis, so promoting mitochondrial FAO (24) or supressing NO inhibition may be useful goals for further work. In addition, FAO is important for maintaining redox homeostasis, by regeneration of NADPH through tricarboxylic acid cycle activity, and hence, may offer protection against EC dysfunction caused by oxidative stress via NOTCH1 regulation (25). Interestingly, supplementation of acetate to increase levels of acetyl-coenzyme A may lead to vasculoprotection against oxidative stress-induced EC dysfunction. Indeed, this concept may also apply to the related process of lymphangiogenesis where $\beta$-oxidation is essential for the development of lymphatic EC, and acetate supplementation may potentiate injury-induced lymphangiogenesis (26). In addition, mitochondrialtargetted peptides may reduce mitochondrial dysfunction and aberrant inflammatory response through activation of nucleotide-binding oligomerisation domain (NOD)like family receptors (27).

\section{Diabetes}

Elevated glucose levels in diabetes lead to an increased expression of insulin receptors and downstream elements of the insulin-signalling pathway (28). A common theme for EC dysfunction begins to emerge where uncoupling of eNOS and increased EC mitochondrial ROS production with hyperglycaemia impair glycolysis by activation of PARP. This may then drive a metabolic realignment in EC towards oxidative phosphorylation $(29,30)$, but in parallel induce EC mitochondriopathy (31). EC ROS production, by activating NADPH-dependent oxidase, leads to EC and vascular smooth muscle dysfunction and oxidative damage. Mitochondrial DNA is sensitive to

This work is licensed under a Creative Commons Attribution-NonCommercial 4.0 International License. 
Nutrition

e.g. hyperglycaemia, caloric restriction

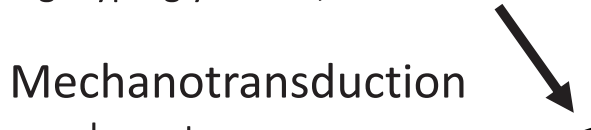

e.g. shear stress

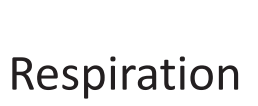

e.g. low $\mathrm{PO}_{2}$

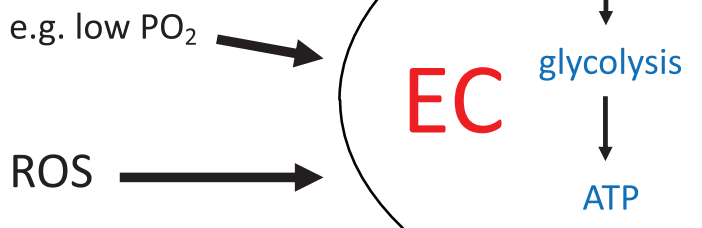

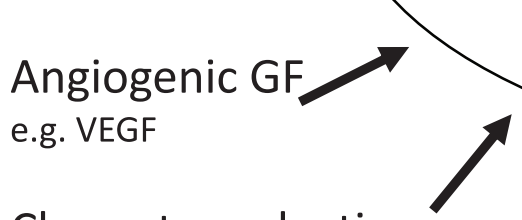

Chemotransduction e.g. cytokines

low mito $\mathrm{V}_{\mathrm{V}} \quad$ complex
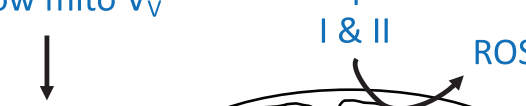

ROS
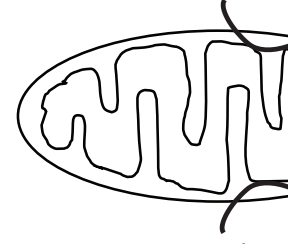

III

VSMC / PC
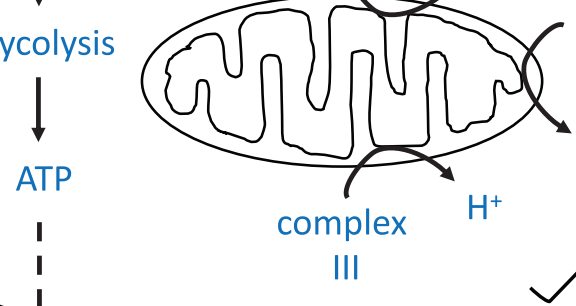

$\beta$ oxidation

$\mathrm{H}^{+}$

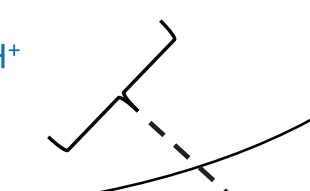

release

$\mathrm{NO}$
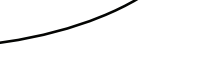

I

\section{EC migration}

Figure 1

Some aspects of mitochondrial biology, illustrating the key findings and concepts relevant to vascular biology, with particular emphasis on endothelial cells and angiogenesis.

modification by ROS, which is observed in cardiovascular disease, smoking, hypercholesterolemia, and obesity (32), suggesting an important therapeutic target.

\section{Tumours}

Tumours require an extensive vascular supply to support high metabolic rates. Thus, starving tumour cells by disrupting their vascular supply provides a rationale for many current therapies. Anti-angiogenesis drugs, for e.g. monoclonal antibodies such as Avastin or receptor tyrosine kinase inhibitors like Sunitinib, are used to target colorectal, non-small-cell lung, ovarian, peritoneal, renal cell and cervical cancers; unfortunately, in most cases treatment provides only a modest improvement in overall patient survival (33). However, reprogramming EC metabolism in the tumour environment may also modulate the excessive angiogenesis (34), as the tumour environment is deprived of nutrients and oxygen, and both tumour and stromal cells must produce large amounts of energy and macromolecular precursors (nucleotides, lipids, amino acids) for rapid growth. EC metabolism and its regulation are therefore centrally involved in vessel growth, survival and function, and may offer novel and alternative targets to interfere with or stimulate angiogenesis. While the hyperglycolytic EC phenotype has been described (35), similar studies identifying mitochondrial targets are lacking.

\section{Drugs}

Various pharmacological studies have been employed to shed important light on the mechanistic role of mitochondrial signalling in coordinating vascular remodelling. This has included the use of small-molecules to activate key upstream mitochondrial molecular regulators (transcription factors or co-factors) including AMPK, PGC-1 $\alpha$, and SIRTUIN (4), although much work is still required to confirm in vivo efficacy. Another major focus has been to use mitochondrial-specific antioxidants (e.g. MitoQ, mito-tempol). Recent work in humans extended experiments performed in rodents (36), showing that chronic oral administration of MitoQ for 6 weeks improved the endothelial functional index of brachial-artery flow-mediated dilatation in healthy older humans (37). While more extensive work is required 
to show whether mitochondrial-specific antioxidant therapies directly impact angiogenesis in humans, initial evidence is promising. It does appear, however, that mitochondrial rather than cytosolic-specific antioxidants (i.e. catalase) provide greater benefits to vascular function (38), supporting the contention that focus in this area should be directed towards mitochondrial-targetted redox signalling. Despite much evidence now indicating ROS as negative regulators of vascular function, antioxidant interventions should be applied with caution given that a minimum level of ROS are required for maintaining normal vascular health. Hence, while acute increases in mitochondrial ROS (e.g. induced via exercise) are key for maintaining normal vascular health, a chronic increase is detrimental as observed during disease.

\section{Conclusions and future directions}

ECs play a key role in supporting angiogenesis and maintaining health; yet, they are predominately fuelled by glycolysis rather than oxidative phosphorylation in which the mitochondria occupy a small cellular volume. However, EC mitochondria are dynamic and adapt to their local environment, such that they provide more energy during proliferation, in response to hypoxia, and when challenged by hyperglycaemia. In recent years it has become clear that one major role for mitochondria in EC is to modulate cellular signalling cascades in response to various stromal cues such as seen with hypoxia, exercise, ischaemia, ageing, and disease (Fig. 1). Thus, contemporary evidence now supports EC mitochondria as major signalling hubs responsible for maintaining vascular homeostasis. One key role seems to be their ability to modulate redox signalling and future avenues should target this pathway as a means of angiogenic therapy. While many questions remain, such as what differences exist between macro- and microvascular EC phenotype, further research on understanding the role of mitochondria in EC beyond energy metabolism will likely yield more effective treatments for modulating angiogenesis in a tissue-specific manner.

\section{Declaration of interest}

The authors declare that there is no conflict of interest that could be perceived as prejudicing the impartiality of this review.

\section{Funding}

This review was supported by British Heart Foundation PG/14/15/30691.

\section{References}

1 Egginton S Physiological factors influencing capillary growth. Acta Physiologica (Oxford, England) 2011202 225-239. (https://doi org/10.1111/j.1748-1716.2010.02194.x)

2 Eelen G, de Zeeuw P, Treps L, Harjes U, Wong BW \& Carmeliet P Endothelial cell metabolism. Physiological Reviews 201898 3-58. (https://doi.org/10.1152/physrev.00001.2017)

3 Flammer AJ, Anderson T, Celermajer DS, Creager MA, Deanfield J, Ganz P, Hamburg NM, Luscher TF, Shechter M, Taddei S, et al. The assessment of endothelial function: from research into clinical practice. Circulation 2012126 753-767. (https://doi.org/10.1161/ CIRCULATIONAHA.112.093245)

4 Kluge MA, Fetterman JL \& Vita JA Mitochondria and endothelial function. Circulation Research 2013112 1171-1188. (https://doi. org/10.1161/CIRCRESAHA.111.300233)

5 Ellsworth ML, Ellis CG \& Sprague RS Role of erythrocyte-released ATP in the regulation of microvascular oxygen supply in skeletal muscle. Acta Physiologica (Oxford, England) 2016216 265-276. (https://doi. org/10.1111/apha.12596)

6 Busija DW, Rutkai I, Dutta S \& Katakam PV Role of mitochondria in cerebral vascular function: energy production, cellular protection, and regulation of vascular tone. Comprehensive Physiology 20166 1529-1548. (https://doi.org/10.1002/cphy.c150051)

7 Caja S \& Enriquez JA Mitochondria in endothelial cells: sensors and integrators of environmental cues. Redox Biology 201712 821-827. (https://doi.org/10.1016/j.redox.2017.04.021)

8 Gutierrez J, Ballinger SW, Darley-Usmar VM \& Landar A Free radicals, mitochondria, and oxidized lipids: the emerging role in signal transduction in vascular cells. Circulation Research 200699 924-932. (https://doi.org/10.1161/01.RES.0000248212.86638.e9)

9 Diebold LP, Gil HJ, Gao P, Martinez CA, Weinberg SE \& Chandel NS Mitochondrial complex III is necessary for endothelial cell proliferation during angiogenesis. Nature Metabolism 20191 158-171. (https://doi.org/10.1038/s42255-018-0011-x)

10 Liu Y, Li H, Bubolz AH, Zhang DX \& Gutterman DD Endothelial cytoskeletal elements are critical for flow-mediated dilation in human coronary arterioles. Medical \& Biological Engineering \& Computing 200846 469-478. (https://doi.org/10.1007/s11517-0080331-1)

11 Al-Mehdi AB, Pastukh VM, Swiger BM, Reed DJ, Patel MR, Bardwell GC, Pastukh VV, Alexeyev MF \& Gillespie MN Perinuclear mitochondrial clustering creates an oxidant-rich nuclear domain required for hypoxia-induced transcription. Science Signaling 20125 ra47. (https://doi.org/10.1126/scisignal.2002712)

12 Franses JW, Drosu NC, Gibson WJ, Chitalia VC \& Edelman ER Dysfunctional endothelial cells directly stimulate cancer inflammation and metastasis. International Journal of Cancer 2013133 1334-1344. (https://doi.org/10.1002/ijc.28146)

13 Ungvari Z, Tarantini S, Kiss T, Wren JD, Giles CB, Griffin CT, Murfee WL, Pacher P \& Csiszar A Endothelial dysfunction and angiogenesis impairment in the ageing vasculature. Nature Reviews. Cardiology 201815 555-565. (https://doi.org/10.1038/s41569-0180030-z)

14 Zhang SX, Wang JJ, Dashti A, Wilson K, Zou MH, Szweda L, Ma JX \& Lyons TJ Pigment epithelium-derived factor mitigates inflammation and oxidative stress in retinal pericytes exposed to oxidized lowdensity lipoprotein. Journal of Molecular Endocrinology 200841 135-143. (https://doi.org/10.1677/JME-08-0011)

15 Moebius-Winkler S, Schuler G \& Adams V Endothelial progenitor cells and exercise-induced redox regulation. Antioxidants \& Redox Signaling 201115 997-1011. (https://doi.org/10.1089/ars.2010.3734)

16 Park SY, Rossman MJ, Gifford JR, Bharath LP, Bauersachs J, Richardson RS, Abel ED, Symons JD \& Riehle C Exercise training improves vascular mitochondrial function. American Journal of Physiology. Heart \& Circulatory Physiology 2016310 H821-H829. (https://doi.org/10.1152/ajpheart.00751.2015) 
17 Gu Q, Wang B, Zhang XF, Ma YP, Liu JD \& Wang XZ Chronic aerobic exercise training attenuates aortic stiffening and endothelial dysfunction through preserving aortic mitochondrial function in aged rats. Experimental Gerontology 201456 37-44. (https://doi. org/10.1016/j.exger.2014.02.014)

18 Valle I, Alvarez-Barrientos A, Arza E, Lamas S \& Monsalve M PGC1alpha regulates the mitochondrial antioxidant defense system in vascular endothelial cells. Cardiovascular Research 200566 562-573. (https://doi.org/10.1016/j.cardiores.2005.01.026)

19 Park SY, Kwon OS, Andtbacka RHI, Hyngstrom JR, Reese V, Murphy MP \& Richardson RS Age-related endothelial dysfunction in human skeletal muscle feed arteries: the role of free radicals derived from mitochondria in the vasculature. Acta Physiologica 2018222. (https://doi.org/10.1111/apha.12893)

20 Davignon J \& Ganz P Role of endothelial dysfunction in atherosclerosis. Circulation 2004109 11127-11132. (https://doi. org/10.1161/01.CIR.0000131515.03336.f8)

21 Wright GL, Maroulakou IG, Eldridge J, Liby TL, Sridharan V, Tsichlis PN \& Muise-Helmericks RC Vegf stimulation of mitochondrial biogenesis: requirement of AKT3 kinase. FASEB Journal 200822 3264-3275. (https://doi.org/10.1096/fj.08-106468)

22 Csiszar A, Labinskyy N, Pinto JT, Ballabh P, Zhang H, Losonczy G, Pearson K, de Cabo R, Pacher P, Zhang C, et al. Resveratrol induces mitochondrial biogenesis in endothelial cells. American Journal of Physiology. Heart \& Circulatory Physiology 2009297 H13-H20. (https:// doi.org/10.1152/ajpheart.00368.2009)

23 Zeng H \& Chen JX Microvascular rarefaction and heart failure with preserved ejection fraction. Frontiers in Cardiovascular Medicine 20196 15. (https://doi.org/10.3389/fcvm.2019.00015)

24 Theodorou K \& Boon RA Endothelial cell metabolism in atherosclerosis. Frontiers in Cell \& Developmental Biology 2018682. (https://doi.org/10.3389/fcell.2018.00082)

25 Kalucka J, Bierhansl L, Conchinha NV, Missiaen R, Elia I, Brüning U, Scheinok S, Treps L, Cantelmo AR, Dubois C, et al. Quiescent endothelial cells upregulate fatty acid $\beta$-oxidation for vasculoprotection via redox homeostasis. Cell Metabolism 201828 881-894.e13. (https://doi.org/10.1016/j.cmet.2018.07.016)

26 Wong BW, Wang X, Zecchin A, Thienpont B, Cornelissen I, Kalucka J, García-Caballero M, Missiaen R, Huang H, Brüning U, et al. The role of fatty acid $\beta$-oxidation in lymphangiogenesis. Nature 2017 542 49-54. (https://doi.org/10.1038/nature21028)

27 Cano Sanchez M, Lancel S, Boulanger E \& Neviere R Targeting oxidative stress and mitochondrial dysfunction in the treatment of impaired wound healing: a systematic review. Antioxidants (Basel) 20187 E98. (https://doi.org/10.3390/antiox7080098)

28 Gosmanov AR, Stentz FB \& Kitabchi AE De novo emergence of insulin-stimulated glucose uptake in human aortic endothelial cells incubated with high glucose. American Journal of Physiology.
Endocrinology \& Metabolism 2006290 E516-E522. (https://doi. org/10.1152/ajpendo.00326.2005)

29 Nishikawa T, Edelstein D, Du XL, Yamagishi S, Matsumura T, Kaneda Y, Yorek MA, Beebe D, Oates PJ, Hammes HP, et al. Normalizing mitochondrial superoxide production blocks three pathways of hyperglycaemic damage. Nature 2000404 787-790. (https://doi.org/10.1038/35008121)

30 Pangare M \& Makino A Mitochondrial function in vascular endothelial cell in diabetes. Journal of Smooth Muscle Research = Nihon Heikatsukin Gakkai Kikanshi 201248 1-26. (https://doi.org/10.1540/ jsmr.48.1)

31 Tang X, Luo YX, Chen HZ \& Liu DP Mitochondria, endothelial cell function, and vascular diseases. Frontiers in Physiology 20145175. (https://doi.org/10.3389/fphys.2014.00175)

32 Knight-Lozano CA, Young CG, Burow DL, Hu ZY, Uyeminami D, Pinkerton KE, Ischiropoulos H \& Ballinger SW Cigarette smoke exposure and hypercholesterolemia increase mitochondrial damage in cardiovascular tissues. Circulation 2002105 849-854. (https://doi. org/10.1161/hc0702.103977)

33 Ribatti D, Annese T, Ruggieri S, Tamma R \& Crivellato E Limitations of anti-angiogenic treatment of tumors. Translational Oncology 2019 12 981-986. (https://doi.org/10.1016/j.tranon.2019.04.022)

34 Bierhansl L, Conradi LC, Treps L, Dewerchin M \& Carmeliet P Central role of metabolism in endothelial cell function and vascular disease. Physiology (Bethesda) 201732 126-140. (https://doi. org/10.1152/physiol.00031.2016)

35 Cantelmo AR, Conradi LC, Brajic A, Goveia J, Kalucka J, Pircher A, Chaturvedi P, Hol J, Thienpont B, Teuwen LA, et al Inhibition of the glycolytic activator PFKFB3 in endothelium induces tumor vessel normalization, impairs metastasis, and improves chemotherapy. Cancer Cell 201630 968-985. (https://doi.org/10.1016/j. ccell.2016.10.006)

36 Gioscia-Ryan RA, LaRocca TJ, Sindler AL, Zigler MC, Murphy MP $\&$ Seals DR Mitochondria-targeted antioxidant (mitoq) ameliorates age-related arterial endothelial dysfunction in mice. Journal of Physiology 2014592 2549-2561. (https://doi.org/10.1113/ jphysiol.2013.268680)

37 Rossman MJ, Santos-Parker JR, Steward CAC, Bispham NZ, Cuevas LM, Rosenberg HL, Woodward KA, Chonchol M, GiosciaRyan RA, Murphy MP, et al. Chronic supplementation with a mitochondrial antioxidant (mitoq) improves vascular function in healthy older adults. Hypertension 201871 1056-1063. (https://doi. org/10.1161/HYPERTENSIONAHA.117.10787)

38 Ungvari Z, Labinskyy N, Gupte S, Chander PN, Edwards JG \& Csiszar A Dysregulation of mitochondrial biogenesis in vascular endothelial and smooth muscle cells of aged rats. American Journal of Physiology. Heart \& Circulatory Physiology 2008294 H2121-H2128. (https://doi.org/10.1152/ajpheart.00012.2008)

Received in final form 29 August 2019

Accepted 1 October 2019

Accepted Preprint published online 1 October 2019 https://vb.bioscientifica.com

https://doi.org/10.1530/VB-19-0018
(C) 2019 The authors Published by Bioscientifica Ltd

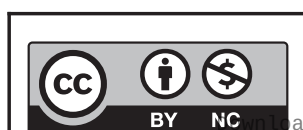

This work is licensed under a Creative Commons Attribution-NonCommercial 4 . O International License.

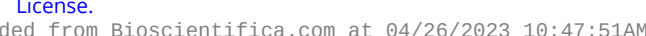
via free access 